\title{
ORIGINAL ARTICLE Development of the hybrid Sleeping Beauty-baculovirus vector for sustained gene expression and cancer therapy
}

\author{
W-Y Luo ${ }^{1}$, Y-S Shih ${ }^{1}$, C-L Hung ${ }^{1}$, K-W Lo ${ }^{1}$, C-S Chiang ${ }^{2}$, W-H Lo ${ }^{1}$, S-F Huang ${ }^{3}$, S-C Wang ${ }^{2}$, C-F Yu ${ }^{2}$, C-H Chien ${ }^{4}$ and Y-C Hu ${ }^{1}$
}

\begin{abstract}
Antiangiogenesis is an appealing anticancer approach but requires continued presence of the antiangiogenic agents, which can be remedied by gene therapy. Baculovirus is an emerging gene delivery vector but only mediates transient expression ( $<7$ days); thus, this study primarily aimed to develop a hybrid baculovirus for sustained antiangiogenic gene expression and cancer therapy. We first constructed plasmids featuring adeno-associated virus inverted terminal repeats (AAV ITRs), oriP/ Epstein-Barr virus-expressed nuclear antigen 1 (EBNA1) or Sleeping Beauty (SB) transposon and compared their efficacies in terms of persistent expression. In human embryonic kidney (HEK293) cells, AAV ITR failed to prolong the expression while oriP/EBNA1 moderately extended the expression to 35 days. In contrast, the SB system led to stable expression beyond 77 days even without antibiotic selection. Given this finding, we constructed a hybrid SB baculovirus expressing the SB transposase and harboring the transgene cassette flanked by inverted repeat/direct-repeat (IR/DR) elements recognizable by SB. The hybrid SB baculovirus efficiently transduced mammalian cells and mediated an expression duration longer than that by conventional baculoviruses, thanks to the transgene persistence and integration. The SB baculovirus (Bac-SB-T2hEA/w) expressing the antiangiogenic fusion protein comprising endostatin and angiostatin ( $\mathrm{hEA}$ ) also enabled prolonged $\mathrm{hEA}$ expression. With sustained hEA expression, Bac-SB-T2hEA/w repressed the angiogenesis in vivo, hindered the growth of two different tumors (prostate tumor allografts and human ovarian tumor xenografts) in mice and extended the life span of animals. These data altogether implicated the potential of the hybrid SB-baculovirus vector for prolonged hEA expression and for the treatment of multiple types of angiogenesis-dependent tumors.
\end{abstract}

Gene Therapy (2012) 19, 844-851; doi:10.1038/gt.2011.129; published online 15 September 2011

Keywords: angiogenesis; baculovirus; cancer therapy; Sleeping Beauty; tumor

\section{INTRODUCTION}

Progression of primary tumors beyond $1-2 \mathrm{~mm}^{3}$ and metastatic tumors critically depend on angiogenesis to supply oxygen and nutrition; ${ }^{1}$ thus, antiangiogenesis represents an appealing anticancer approach. For instance, endostatin and angiostatin are potent angiogenesis inhibitors and can inhibit tumor growth in numerous animal tumor models. ${ }^{1}$ However, the antiangiogenic protein must be maintained at a level sufficient to offset the secretion of proangiogenic stimuli, thereby entailing repeated protein administrations. ${ }^{2}$ This problem may be alleviated by the expression of the antiangiogenic gene via such vectors as adenovirus, adeno-associated virus (AAV) or vaccinia virus. ${ }^{3}$ It was shown that the oncolytic adenovirus expressing a human endostatin and angiostatin fusion protein (hEA) imposed potent antiangiogenic effects and suppressed the prostate tumor growth in mouse models, ${ }^{4}$ while the vaccinia virus expressing hEA also delayed the pancreatic cancer progression. ${ }^{5}$ However, these vectors possess respective drawbacks. ${ }^{3,6}$ For instance, adenovirus mounts potent immune responses while the packaging capacity of AAV is limited.

Baculovirus is an insect virus yet it also efficiently transduces a broad range of mammalian cells with minimal cytotoxicity. Baculovirus is non-pathogenic to humans and recombinant baculovirus construction and handling can be performed in Biosafety Level 1 facilities. Consequently, baculovirus has emerged as a promising gene delivery vector for a plethora of applications (for review, see Chen et al., ${ }^{7}$ Madhan et al., ${ }^{8}$ Kost et al., ${ }^{9}$ Liu et al., ${ }^{10}$ Lin et al., ${ }^{11}$ and Airenne et al. ${ }^{12}$ ). Furthermore, baculovirus has been exploited for the treatment of glioma, hepatoma and melanoma in murine models. ${ }^{13-15}$ The baculovirus expressing the herpes simplex virus thymidine kinase repressed the growth of human glioblastoma in the presence of ganciclovir and prolonged the mouse survival; yet, the tumors were not completely regressed partly due to the transient herpes simplex virus thymidine kinase expression. ${ }^{16}$ To combat the prostate cancer, we constructed a baculovirus expressing hEA (Bac-hEA) and proved the antiangiogenic functions. Intratumoral injection of Bac-hEA into the prostate tumor grafts in mice attenuated the angiogenesis and tumor growth and extended the life span of animals. ${ }^{17}$ However, the baculovirus-mediated hEA expression was transient and the antitumor effect was temporary. Since antiangiogenic gene therapy is directed toward inhibiting the tumor neovasculaturization rather than toward the tumor cells, it is critical to confer stable expression of the antiangiogenic factors at therapeutic levels. ${ }^{18}$ As such, developing a new baculovirus vector capable of long-term expression is desired to ameliorate and prolong the antitumor effects.

Baculovirus mediates short-term expression as a result of the non-replication and non-integrating nature of baculovirus within the transduced cells. ${ }^{19,20}$ Currently, a number of systems,

${ }^{1}$ Department of Chemical Engineering, National Tsing Hua University, Hsinchu, Taiwan; ${ }^{2}$ Department of Biomedical Engineering and Environmental Sciences, National Tsing Hua University, Hsinchu, Taiwan; ${ }^{3}$ Division of Molecular and Genomic Medicine, National Health Research Institutes, Miaoli, Taiwan and ${ }^{4}$ Institute of Biochemistry and Molecular Biology, School of Life Science, National Yang-Ming University, Taipei, Taiwan. Correspondence: Professor Y-C Hu, Department of Chemical Engineering, National Tsing Hua University, 101, Section 2, Kuang Fu Road, Hsinchu 300, Taiwan.

E-mail: ychu@mx.nthu.edu.tw

Received 18 May 2011; revised 29 July 2011; accepted 8 August 2011; published online 15 September 2011 
including the oriP/Epstein-Barr virus-expressed nuclear antigen 1 (EBNA1), AAV inverted terminal repeats (ITRs) and Sleeping Beauty (SB) transposon, are available to maintain the transgene in an episomal or integrated form. oriP is an origin of replication derived from Epstein-Barr virus, which can be recognized by EBNA1, leading to the replication and subsequent segregation of episomal Epstein-Barr virus DNA; ${ }^{21}$ hence, constructs containing oriP/EBNA1 have been exploited for persistent expression. ${ }^{22,23}$ The AAV ITR system consists of the transgene cassette flanked by the AAV left and right ITR, which can be recognized by the AAV Rep proteins for site-specific integration into the host chromosome. ${ }^{24}$ Conversely, the SB system comprises the SB transposase and the genetic cargo framed by the terminal inverted repeats/directrepeat (IRs/DR), whereby the SB transposase binding to the IR/DR sequences results in the transposon excision from the donor and subsequent integration into the chromosome (cut-and-paste mechanism) ${ }^{25}$ The SB system has been used in conjunction with adenovirus $^{26}$ or herpes simplex virus amplicon ${ }^{27}$ to generate hybrid vectors for prolonged expression.

Despite the promise of these three systems, direct comparison of these systems with regard to long-term expression is missing. Therefore, we first compared the performance of these three systems for persistent expression in mammalian cells and uncovered that the SB system most effectively extended the expression. Consequently, we generated the hybrid SB baculovirus and examined the gene integration and the prolonged expression of enhanced green fluorescent protein (EGFP). The hybrid SB baculovirus was further assessed for the ability to confer prolonged hEA expression and impart antiangiogenic and antitumor effects in the prostate cancer model. The ability of the hEA-expressing SB-baculovirus vector to suppress the ovarian tumor xenografts in mice was also evaluated.

\section{RESULTS}

Comparison of oriP/EBNA1, AAV ITR and SB systems

To compare the efficacy in long-term transgene expression, we first constructed a series of plasmids that featured one of the three systems (oriP/EBNA1, AAV ITR and SB) and harbored the CMVEGFP cassette (Figure 1a). pBac-CE encompassed the CMV-EGFP cassette and served as the control. With pBac-CE as the backbone, pBac-COE-CE accommodated an additional cassette comprising oriP and the EBNA1 gene driven by the CMV-IE promoter, so that the expressed EBNA1 could interact with oriP and facilitate the plasmid replication/segregation. Conversely, pBac-ITR-CE encompassed the CMV-EGFP cassette flanked by the AAV ITR elements to exploit the AAV ITR system (Figure 1a). Since the AAV genome integration necessitates Rep proteins, pBac-ITR/Rep-CE was prepared based on pBac-ITR-CE to accommodate an additional Rep expression cassette driven by its own endogenous promoter. Moreover, pBac-SB-T2CE harbored a CMV-SB expression cassette along with the IR/DR-flanking CMV-EGFP cassette (transposon), hoping that the expressed SB could transpose the IR/DR-flanking cassette to the chromosome.

These plasmids $\left(3 \mu \mathrm{g}\right.$ per $5 \times 10^{5}$ cells) were separately transfected into human embryonic kidney (HEK293) cells, which were cultured and passaged for total fluorescence intensity (FI) (expressed in arbitrary units (a.u.)) measurements by flow cytometry. Figure $1 \mathrm{~b}$ shows that pBac-CE and pBac-ITR-CE only led to transient EGFP expression, which plummeted after day 5 and dropped below the detection limit $\left(\approx 10^{5}\right.$ a.u. $)$ at day 14 . Surprisingly, pBac-ITR-CE/Rep also failed to extend the expression duration despite the inclusion of the rep gene. pBac-COE-CE remarkably prolonged the expression which, however, extinguished after day 35. Only pBac-SB-T2CE led to stable EGFP expression, which remained relatively constant and persisted for at least 77 days.
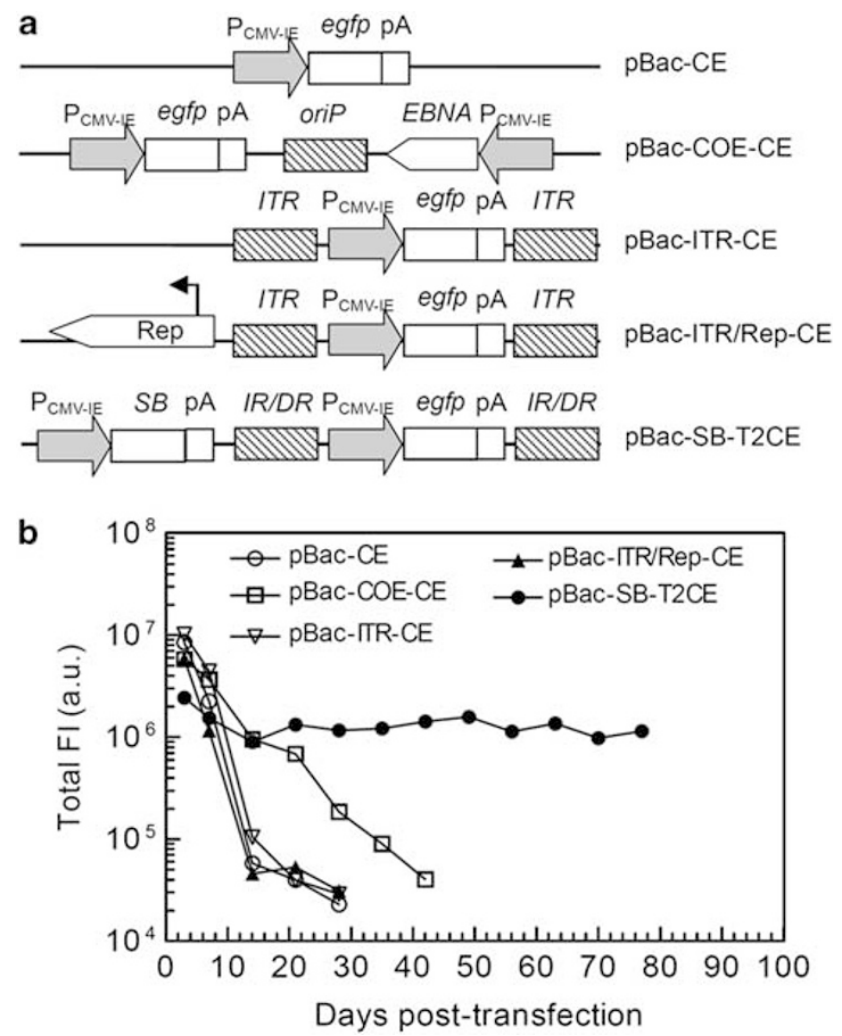

Figure 1. Comparison of expression durations mediated by different systems. (a) Schematic illustrations of the plasmids. (b) Gene expression profiles. HEK293 cells were transfected with equal amounts of the plasmids, continued to be cultured, passaged and analyzed by flow cytometry. The total Fls were yielded by multiplying the number of GFP + cells and mean Fl, and are expressed in a.u. The data represent the average total $\mathrm{Fl}$ of three independent culture experiments.

The hybrid SB baculovirus resulted in transgene integration and prolonged expression

Since the SB system (pBac-SB-T2CE) gave rise to the most stable expression, we selected $\mathrm{pBac}-\mathrm{SB}-\mathrm{T} 2 \mathrm{CE}$ for the construction of the hybrid baculovirus Bac-SB-T2CE. HEK293 cells were mock transduced or transduced with Bac-SB-T2CE and continued to be cultured in the absence of antibiotic selection. The flow cytometry analyses (Figure 2a) demonstrated that Bac-SB-T2CE transduced HEK293 cells at efficiencies up to $96 \%$ at 2 days post-transduction (d.p.t.). At 24 d.p.t., $\approx 42 \%$ of the transduced cells remained fluorescing (Figures $2 \mathrm{a}$ and $\mathrm{b}$ ).

Concomitantly, the PCR analyses using primer sets specific for egfp and two fragments on the baculovirus genome (BM3 and BM4, upper panel in Figure 2c) depicted that all three gene fragments were present in the purified baculoviral genome (positive control) but were absent in the mock-transduced cells (Figure 2c). In the transduced cells, the egfp gene was evidently present at 2 d.p.t. and remained detectable at 24 d.p.t., attesting the persistence of the transgene. In contrast, the viral gene fragments (BM3 and BM4) were only detectable at 2 d.p.t. but disappeared at 24 d.p.t., agreeing with the notion that baculoviral DNA is degraded and diluted within the mammalian cells. ${ }^{28}$

To verify the state of the DNA, fluorescent in situ hybridization (FISH) experiment using the egfp probe was performed at 24 d.p.t. Figure $2 \mathrm{~d}$ illustrates the presence of a significant number of bright fluorescent spots associated with the chromosomes. In contrast, no fluorescent spots were associated with the chromosome in the cells transduced with an egfp-bearing baculovirus deficient in the 
a
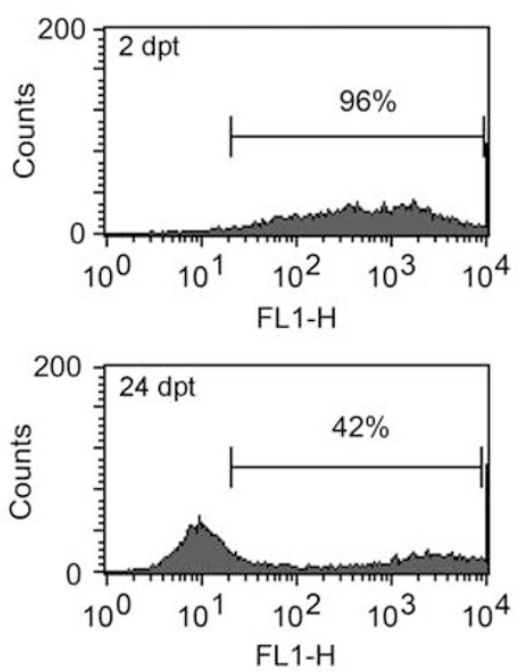

C IR/DR $\mathrm{P}_{\text {CMV.JE }}$ egfp pA IR/DR
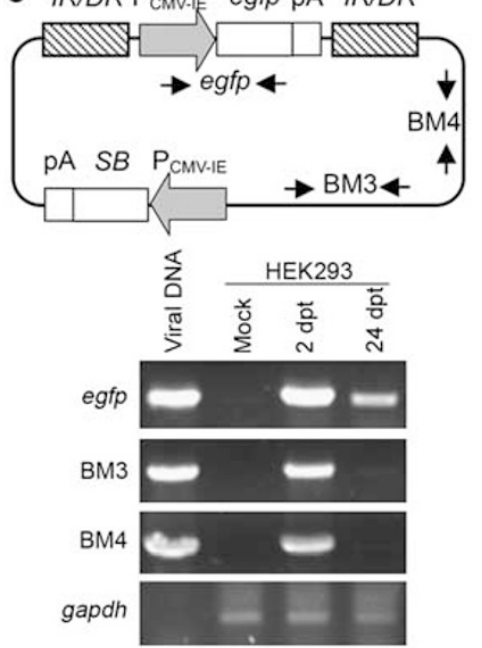

b

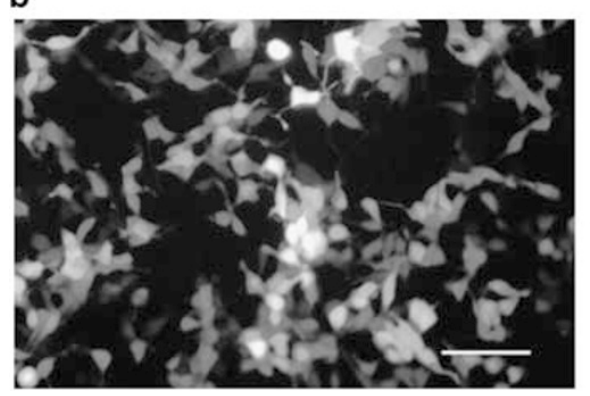

d



Figure 2. The hybrid SB-baculovirus Bac-SB-T2CE conferred prolonged expression, thanks to transgene integration. (a) Percent of GFP + cells as measured by flow cytometry. (b) Microscopic observation of fluorescence emitting cells (bar, $100 \mu \mathrm{m}$ ). (c) Transgene persistence as confirmed by PCR. (d) Transgene integration as confirmed by FISH. HEK293 cells were transduced with Bac-SB-T2CE and analyzed by flow cytometry and PCR at 2 and 24d.p.t., or alternatively subjected to fluorescence microscopy and FISH analyses at 24 d.p.t. The PCR was performed using primers specific for egfp and two fragments (BM3 and BM4) on the baculoviral genome. The data are representative of three independent culture experiments.

IR/DR elements and CMV-SB cassette. ${ }^{20}$ Figure 2 thus collectively indicated that the transgene persistence stemmed from gene integration into the host chromosome, thanks to the SB-mediated transposition.

The hybrid SB baculovirus conferred prolonged hEA expression Given that hEA retards angiogenesis and suppresses tumor progression, we next constructed Bac-SB-T2hEA/w, which accommodated both the $S B$ gene and the IR/DR-flanking $h E A$ gene (Figure 3a). As controls, we also prepared Bac-T2-hEA/w that carried only the $h E A$ gene and Bac-SB-T2luc/w, which harbored the $\mathrm{SB}$ transposase gene and the IR/DR-flanking luciferase gene.

To confirm the sustained hEA expression, HEK293 cells were transduced with Bac-T2hEA/w or Bac-SB-T2hEA/w and the supernatants were collected upon subculture for ELISA. Figure $3 b$ delineates that both Bac-T2hEA/w and Bac-SB-T2hEA/w effectively expressed hEA at 2 d.p.t. Without the aid of SB, the Bac-T2hEA/wmediated hEA expression precipitously dropped to $\approx 20 \mathrm{ng} \mathrm{ml}^{-1}$ at 14 d.p.t. In contrast, the hEA expression conferred by Bac-SBT2hEA/w declined initially but remained stably higher than $200 \mathrm{ng} \mathrm{ml}^{-1}$ at 14 d.p.t, attesting that baculovirus coupled with the SB system prolonged the hEA expression.

The hybrid SB baculovirus conferred prolonged antitumor effects To evaluate the antitumor effects imparted by Bac-SB-T2hEA/w, the virus was injected intratumorally $\left(3 \times 10^{9}\right.$ plaque forming units (p.f.u.) per mouse) into the mice bearing prostate tumors
( $n=6$ per group) when the tumor volume reached $\approx 70 \mathrm{~mm}^{3}$. As controls, phosphate-buffered saline (PBS) and Bac-SB-T2luc/w were injected into the tumors in a similar manner, respectively.

Figure 4 a depicts that the tumor size in the PBS group quickly grew to $>500 \mathrm{~mm}^{3}$ at 9 days post-injection. Albeit the inclusion of the CMV-SB cassette and IR/DR-flanking luciferase cassette, Bac-SB-T2luc/w injection slightly suppressed the tumor growth but the tumor volume continued to increase and approached $\approx 500 \mathrm{~mm}^{3}$ at day 12 . In contrast, Bac-SB-T2hEA/w injection restricted the tumor size to $<100 \mathrm{~mm}^{3}$ for 18 days and led to the tumor regression in one mouse at day 21 . The tumor growth in other mice continued after day 18 but at a markedly lower rate when compared with those of the Bac-SB-T2luc/w and PBS groups, and the average tumor volume did not reach $500 \mathrm{~mm}^{3}$ until day 33. Consistent with the tumor volume data, the life span (median survival) of the mice was prolonged $(P<0.005)$ from 9 days for the PBS group to 12 days for the Bac-SB-T2luc/w group (Figure 4b). Bac-SB-T2hEA/w injection further extended the life span to 33 days $(P<0.005$ when compared with the Bac-SB-T2luc/w group), confirming that Bac-SB-T2hEA/w effectively mitigated the prostate tumor growth.

Whether the antitumor effects arose from the repression of angiogenesis was examined by repeating the in vivo experiments, followed by mice kill when the tumor volume reached $200 \mathrm{~mm}^{3}$. CD31-specific immunohistochemical staining of the tumor sections (Figure 5a) illustrated apparent formation of branching vessels within the tumors treated with PBS, indicating the occurrence of angiogenesis that accompanied the tumor growth. 
a
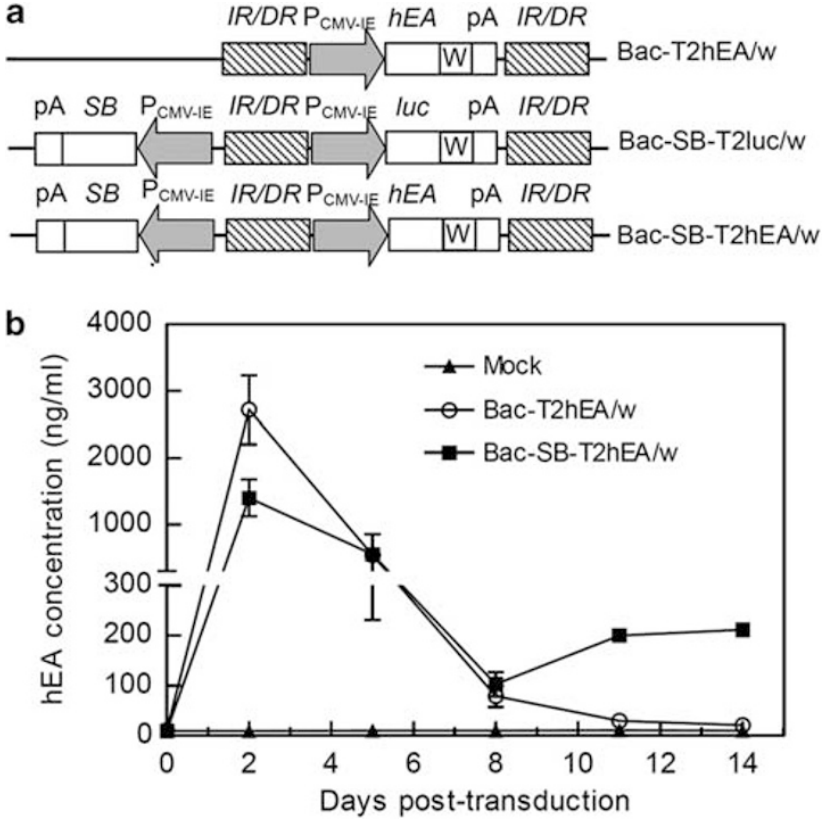

Figure 3. The hybrid SB-baculovirus Bac-SB-T2hEA/w prolonged the hEA expression. (a) Schematic illustration of the baculovirus constructs. (b) hEA expression profile. HEK293 cells were transduced with Bac-T2hEA/w (deficient in SB expression) or Bac-SB-T2hEA/w and continued to be subcultured every 3 days. The supernatant was collected at different times for hEA expression analyses. W denotes WPRE, a cis-acting element that enhanced the baculovirus-mediated transgene expression in vertebrate cells.
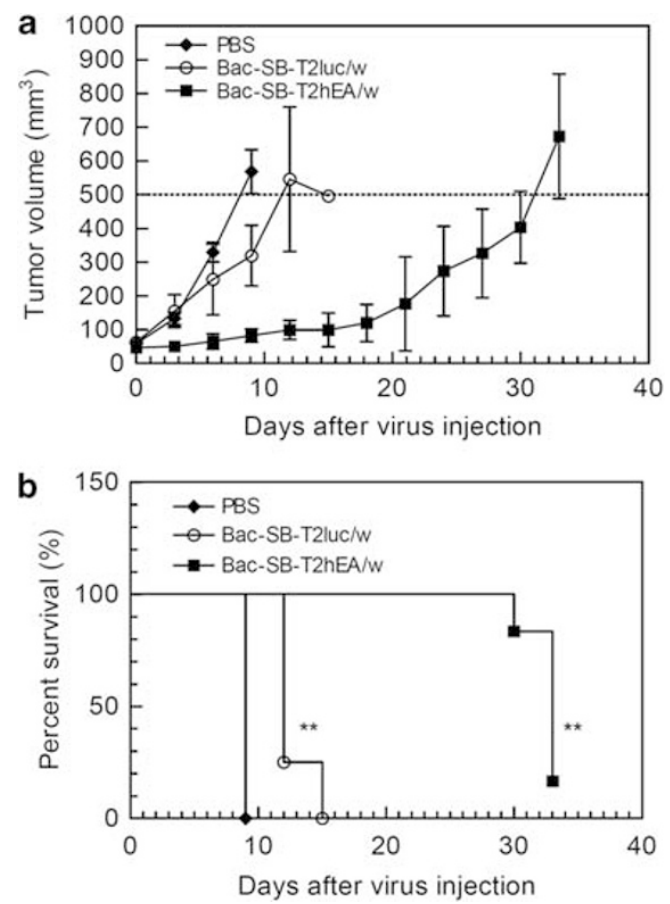

Figure 4. Bac-SB-T2hEA/w conferred antitumor effects in mouse prostate cancer models. (a) Tumor volume. (b) Percent survival. Mouse TRAMP-C1 cells $\left(1 \times 10^{6}\right.$ cells per mouse) were injected subcutaneously into the dorsal side of C57BL/6J mice ( $n=6$ for each group). When the tumor volume reached $70 \mathrm{~mm}^{3}$, PBS, Bac-SBT2luc/w or Bac-SB-T2hEA/w was injected intratumorally at days 0,3 and 6. ${ }^{* *}$ Indicates the significant difference $(P<0.005)$ between the Bac-SB-T2luc/w and Bac-SB-T2hEA/w groups. a
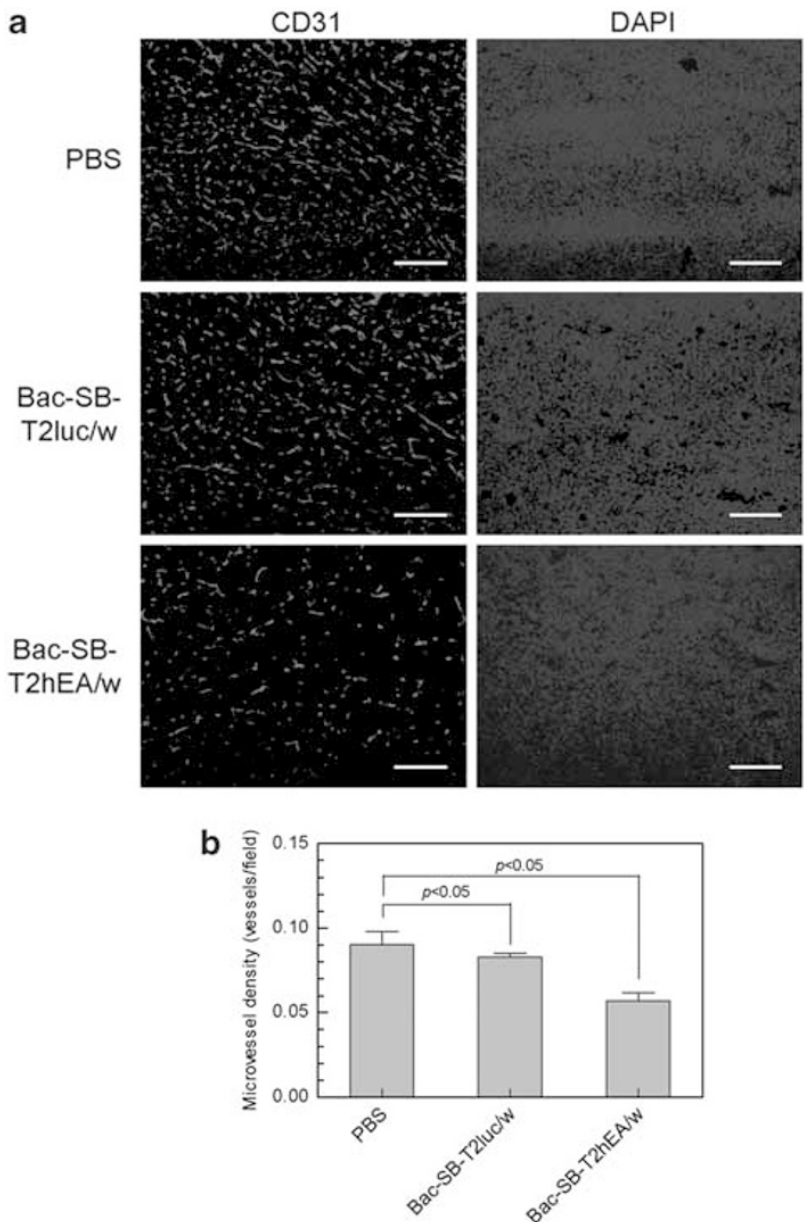

Figure 5. Bac-SB-T2hEA/w conferred antiangiogenesis effects in mouse prostate cancer models. (a) CD31-specific immunohistochemical staining. (b) Quantitative analyses of microvessel density. Tumor specimens were removed when tumor volume reached $200 \mathrm{~mm}^{3}$, sectioned and subjected to CD31-specific immunohistochemical staining. The microvessel density was calculated in five random fields within the tumor area using Image Pro Plus 6.0 by dividing the CD31-positive pixels by the total pixels. Bar, $100 \mu \mathrm{m}$.

Bac-SB-T2luc/w only marginally attenuated the vessel formation. In line with the retarded tumor growth, Bac-SB-T2hEA/w injection led to distinctly less organized and scarce microvasculature structures within the tumors (Figure 5a). Quantitative analysis of the microvessel densities (Figure $5 b$ ) further confirmed the more significant $(P<0.05)$ inhibition of angiogenesis by Bac-SB-T2hEA/w than by PBS or Bac-SB-T2luc/w.

To explore whether Bac-SB-T2hEA/w was also able to hinder the growth of another tumor type, the virus $\left(3 \times 10^{9}\right.$ p.f.u. per mouse) or PBS was injected into ovarian tumor xenografts when the tumor volume reached $\approx 70 \mathrm{~mm}^{3}$ ( $n=4$ per group). Figure $6 \mathrm{a}$ depicts that the average tumor size in the PBS group grew to $>500 \mathrm{~mm}^{3}$ at 36 days post-injection. Bac-SB-T2luc/w injection delayed the tumor growth and the tumor volume approached $\approx 500 \mathrm{~mm}^{3}$ at day 54 . Of note, Bac-SB-T2hEA/w strikingly inhibited the tumor development, with the size increasing at a rate considerably lower than those of the Bac-SB-T2luc/w and PBS groups. At the end of experiments (90 days after virus injection), the average tumor volume remained $<200 \mathrm{~mm}^{3}$ and none of the tumors grew to $>300 \mathrm{~mm}^{3}$ in size. Concurrently, the life span of the mice in the Bac-SB-T2hEA/w group was dramatically $(P<0.005)$ prolonged (Figure 6b). These data collectively 

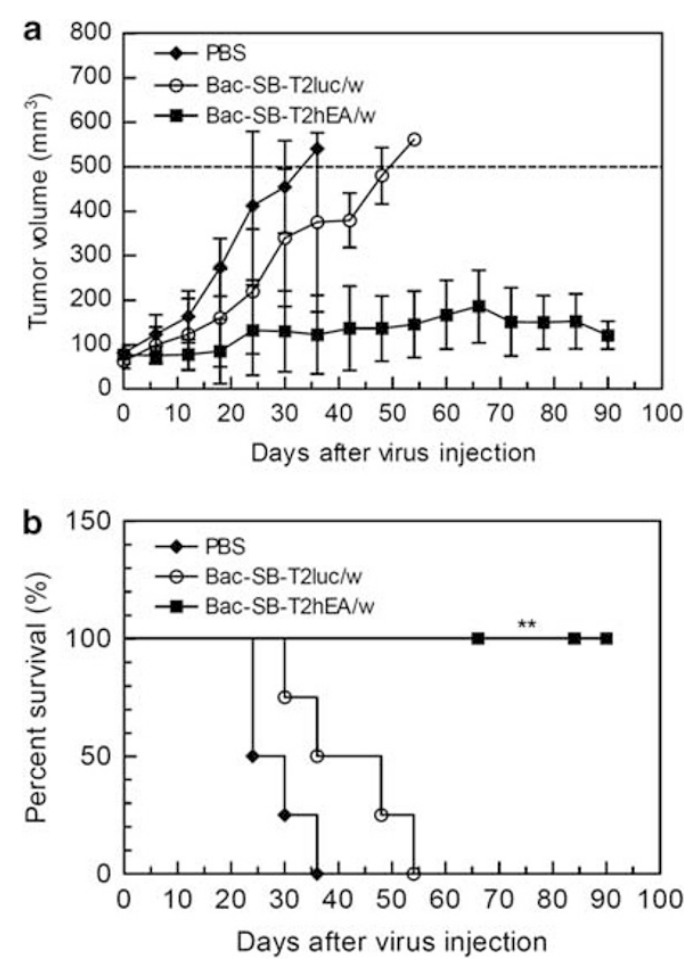

Figure 6. Bac-SB-T2hEA/w conferred antitumor effects in human ovarian tumor xenografts. (a) Tumor volume. (b) Percent survival. Human SKOV3 cells $\left(1 \times 10^{6}\right.$ cells per mouse) were injected subcutaneously into the back of BALB/c nu/nu mice $(n=4$ for each group). When the tumor volume reached $70 \mathrm{~mm}^{3}, \mathrm{PBS}, \mathrm{Bac}-\mathrm{SB}-$ T2luc/w or Bac-SB-T2hEA/w was injected intratumorally at days 0,3 and 6. ${ }^{* *}$ Indicates the significant difference $(P<0.005)$ between the Bac-SB-T2luc/w and Bac-SB-T2hEA/w groups.

confirmed that Bac-SB-T2hEA/w was effective in suppressing the ovarian tumor growth.

\section{DISCUSSION}

The overriding objective of this study was to develop a hybrid baculovirus vector for prolonged antiangiogenic gene expression and cancer therapy. Since head-to-head comparison of AAV ITR, oriP/EBNA1, and SB systems with respect to long-term expression was lacking, we first evaluated which one of the three systems most effectively prolonged the transgene expression. To our surprise, sandwiching the transgene cassette with the AAV ITR elements (pBac-ITR-CE) and inclusion of the rep gene in cis (pBac-ITR/ Rep-CE) failed to prolong the expression (Figure 1b). Attempts to supply the rep gene in trans (by co-delivering the rep gene in another construct with the ITR-flanking CMV-EGFP construct) were also unable to extend the expression duration (data not shown). These observations contradicted with previous data reporting that the transgene cassette flanked by the ITR elements, with or without the rep gene, offered sustained expression in the rat brains (>90 days), ${ }^{24}$ in human neuronal cells ${ }^{29}$ and in human embryonic stem cells ( $>22$ weeks). ${ }^{30}$ The disparity probably arose from the differences in the intrinsic properties (for example, repertoire of cellular factors) or in the proliferation rates of the cell types. Should integration not occur efficiently, the ITR-flanking cassette was more likely to persist in the slow proliferating neuronal/stem cells for a longer period of time than in the rapidly proliferating HEK293 cells in which the transgene was diluted out. These data also suggested the limitation of the AAV ITR system.

Conversely, the oriP/EBNA1 system (pBac-COE-CE) prolonged the gene expression to 35 days yet the expression decayed with time without antibiotic selection. The declining expression was observed previously ${ }^{22,31}$ and was attributable to the inability of oriP/EBNA1 to warrant $100 \%$ plasmid replication and segregation to daughter cells in each cell cycle, ${ }^{31}$ which led to progressive dilution of the transgene within the cells upon growth and subculture.

Among these three systems, only the SB system (pBac-SB-T2CE) successfully gave rise to stable expression for at least 77 days, even without the selective pressure (Figure 1b). In light of this finding, we further constructed the hybrid SB baculovirus and confirmed the efficient transduction, prolongation of transgene expression (Figures $2 \mathrm{a}$ and $\mathrm{b}$ ) and the transgene persistence within the cells (Figure 2c). Since SB-mediated transposition adopts a cut-and-paste mechanism ${ }^{32}$ and DNA-binding enzyme (for example, the flippase recombination enzyme) is able to recognize target sequences on the baculovirus and cleave the transgene off the baculovirus genome, ${ }^{22}$ it is reasonable to envisage that the expressed SB transposase excised the genetic cargo framed by the IR/DR sequences, leading to the transgene integration as evidenced by FISH (Figure 2d).

Due to the integration, the hybrid SB-baculovirus vectors allowed for prolonged expression of EGFP (Figure 2a) and hEA (Figure $3 \mathrm{~b}$ ), whose durations considerably outlasted those of the conventional baculovirus vectors (typically $<7$ days in dividing cells ${ }^{33}$ ). The Bac-SB-T2hEA/w-mediated hEA expression was robust initially (for example, at 2 d.p.t.) as a result of the abundant episomal transgenes introduced by the SB-baculovirus vectors; yet, the expression plummeted because the transgene copies that failed to integrate underwent degradation and dilution upon cell subculture. ${ }^{23}$ Nevertheless, the hEA expression (Figure $3 \mathrm{~b}$ ) became steady after day 11 at a level $\left(212 \mathrm{ng} \mathrm{ml}^{-1}\right)$ significantly higher than that mediated by the SB-deficient baculovirus (Bac-T2hEA/w). The stable hEA level was attributable to the expression from the integrated transgene and was $\approx 13 \%$ that of the peak level at day $2\left(1572 \mathrm{ng} \mathrm{ml}^{-1}\right)$, which might reflect the transposition efficiency and agreed with the efficiency for human peripheral blood lymphocytes. ${ }^{34}$ Recently, a hyperactive SB100X transposase was developed, which yielded more efficient transposition efficiencies $\left(\approx 35-50 \%\right.$ in human CD34 + cells $\left.^{35}\right)$ than SB11 used in this study, and supported long-term ( $>1$ year) transgene expression both in vitro and in vivo. ${ }^{35,36}$ Our preliminary data also showed that co-injection of an SB100X-expressing baculovirus and another baculovirus harboring the IR/DR-flanking luciferase gene into the mice conferred stable expression in vivo for $>86$ days (Supplementary Figure S1). Therefore, use of SB100X in lieu of SB11 in the future should ameliorate the transposition efficiency and yield more robust and stable transgene expression.

With sustained hEA expression, Bac-SB-T2hEA/w was able to impede the growth of prostate tumor grafts (Figure 4a) and extend the life span of the mice (Figure 4b). The antitumor effects were significantly superior to those mediated by the control viruses Bac-SB-T2luc/w (Figure 4) and Bac-hEA (a baculovirus transiently expressing hEA; Supplementary Figure S2), and concurred with the blockade of new blood vessel formation within the tumors (Figure 5). Furthermore, Bac-SB-T2hEA/w administration inhibited the growth of the ovarian tumor xenografts (Figure 6a) and remarkably elevated the percent survival of mice (Figure 6b). Although the persistent hEA expression in vivo was not confirmed due to the lack of ELISA kits for reliable measurements of $\mathrm{hEA}$ in the sera, these data collectively attested that Bac-SB-T2hEA/w capable of prolonged $\mathrm{hEA}$ expression could attenuate the angiogenesis and augment the antitumor effects for the treatment of multiple types of tumors.

Another beneficial determinant to the antitumor efficacy might be the innate immune responses elicited by baculovirus, which included the induction of such cytokines as interferon $\alpha / \beta$, interleukin- 6 , interleukin- 8 , interleukin- $1 \beta$ and tumor necrosis 
factor- $\alpha^{37-39}$ as well as activation of macrophages ${ }^{40}$ and natural killer cells. ${ }^{13}$ These responses altogether conferred the animals the antitumor immunity as reported previously ${ }^{41}$ and as evidenced by the slightly hindered tumor progression (Figures $4 a$ and $6 a$ ) by the control virus Bac-SB-T2luc/w. These properties rendered baculovirus a powerful adjuvant to potentiate the antitumor efficacies.

Taken together, we developed a hybrid SB-baculovirus vector for efficient mammalian cell transduction and sustained transgene expression. Unlike gammaretrovirus and lentivirus that have the propensity to integrate the transgenes into the transcription start sites or transcription units, ${ }^{42}$ the SB transposon results in fairly unbiased genomic integration, which reduces the risk of insertional mutagenesis and has been coupled with the lentiviral vector to redirect the vector integration preference. ${ }^{43}$ Moreover, SB seems to trigger significantly milder epigenetic changes at the genomic insertion site, hence minimizing the potential genotoxicity. ${ }^{36}$ The SB-baculovirus vector took advantage of the efficient baculovirus transduction, alleviated the shortcoming of conventional baculovirus vectors (that is, transient expression) and augmented the antitumor effects by expressing the antiangiogenic hEA protein. It should be noted, however, that some tumors can obtain vascular supply though other mechanisms such as vessel cooption and upregulation of alternative proangiogenic pathways. $^{2}$ As such, combination therapy whereby Bac-SB-T2hEA/ w coupled with other therapies (for example, radiotherapy or chemotherapy) ought to exert more potent antitumor efficacies. ${ }^{44}$

\section{MATERIALS AND METHODS}

\section{Cells and media}

HEK293 cells were cultured in DMEM medium (Sigma, St Louis, MO, USA) containing $10 \%$ fetal bovine serum (Hyclone, Logan, UT, USA). Mouse prostate cancer cells (TRAMP-C1) were cultured in DMEM medium containing $10 \%$ fetal bovine serum, $5 \mu \mathrm{g} \mathrm{ml}^{-1}$ bovine insulin (Sigma) and $1 \times 10^{-8} \mathrm{M}$ dihydrotestosterone (Sigma). Human ovarian cancer cells SKOV3 were cultured in McCoy's 5A medium (Sigma) supplemented with $15 \%$ fetal bovine serum.

\section{Preparation of recombinant baculoviruses}

The plasmid pBac-CE was constructed previously and harbored the reporter gene egfp under the CMV-IE promoter (CMV-EGFP cassette). ${ }^{28}$ The CMV-EGFP cassette was also PCR amplified from pEGFP-N1 (Clontech, Mountain View, CA, USA) and subcloned into the oriP/EBNA1-containing pRep4 (Invitrogen, Carlsbad, CA, USA). The cassette comprising CMV-EGFP and oriP/EBNA1 was then subcloned into pFastBac $\Delta$ polh $\Delta \mathrm{p} 10$, whose polyhedrin and p10 promoters were removed from pFastBac DUAL ${ }^{20}$ to yield $\mathrm{pBac}-\mathrm{COE}-\mathrm{CE}$. To generate $\mathrm{pBac}-\mathrm{ITR}-\mathrm{CE}$, the egfp gene from pEGFP-N1 was PCR amplified and subcloned into pAAV-MCS (Stratagene, Santa Clara, CA, USA) in between the CMV-IE promoter and the polyadenylation signal (pA). The CMV-EGFP cassette was then subcloned into pBac-AAV-lac $Z^{45}$ to replace the AAV ITR-flanking CMV-lacZ cassette, so that the resultant pBacITR-CE accommodated the CMV-EGFP cassette flanked by ITR. To construct $\mathrm{pBac}-\mathrm{ITR} / \mathrm{Rep}-\mathrm{CE}$, the rep (rep68/78) gene was amplified from PAAV-RC (Stratagene) and subcloned into pBac-ITR-CE.

In parallel, the gene encoding the SB11 transposase was digested from pCMV-SB (kindly provided by Professor PB Hackett, University of Minnesota) by EcoRI/Sall treatment and subcloned into pFastBac $\Delta$ polh $\Delta \mathrm{p} 10$ to yield $\mathrm{pBac}-\mathrm{SB}$. The left and right IR/DR elements were amplified from pT2-BH (a gift from Professor PB Hackett) by Sall/Notl digestion and cloned into pBac-SB to yield pBac-SB-T2. The CMV-EGFP cassette was subcloned into $\mathrm{pBac}-\mathrm{SB}-\mathrm{T} 2$ in between the IR/DR elements to yield the resultant $\mathrm{pBac}-\mathrm{SB}-\mathrm{T} 2 \mathrm{CE}$.

The $h E A$ fusion gene was PCR amplified from pBLAST42-hEndo::Angio (InvivoGen, San Diego, CA, USA) and cloned into pBac-CE by Notl/Sall treatment to generate pBac-hEA. The WPRE (woodchuck hepatitis virus post-transcriptional regulatory element), a cis-acting element that enhanced the baculovirus-mediated transgene expression in vertebrate cells, ${ }^{46}$ was amplified from pFastBac1-CMV-EGFP-WPRE ${ }^{30}$ (kindly provided by Professor Shu Wang of National University of Singapore) and inserted into $\mathrm{pBac}-\mathrm{hEA}$ between the $h E A$ gene and $\mathrm{pA}$ to yield pBac-hEA/w. The IR/ $\mathrm{DR}$ elements from $\mathrm{pT} 2-\mathrm{BH}$ were digested by Sall/Notl and cloned into pFastBac $\Delta$ polh $\Delta$ p10 to yield pBac-T2. The CMV-hEA-WPRE cassette was then amplified from $\mathrm{pBac}-\mathrm{hEA} / \mathrm{w}$ and subcloned into $\mathrm{pBac}-\mathrm{T} 2$ and $\mathrm{pBac}-$ $\mathrm{SB}-\mathrm{T} 2$ to yield $\mathrm{pBac}-\mathrm{T} 2 \mathrm{hEA} / \mathrm{w}$ and $\mathrm{pBac}-\mathrm{SB}-\mathrm{T} 2 \mathrm{hEA} / \mathrm{w}$, respectively. $\mathrm{pBac}-\mathrm{SB}-$ T2luc/w was constructed by amplifying the luciferase (luc) gene from pCMV-Gluc (New England BioLabs, Ipswich, MA, USA) and replacing the $h E A$ gene in $\mathrm{pBac}-\mathrm{SB}-\mathrm{T} 2 \mathrm{hEA} / \mathrm{w}$ by AsiSI/Asull treatment.

pBac-SB-T2CE, pBac-T2hEA/w, pBac-SB-T2luc/w and pBac-SB-T2hEA/w were used to construct the recombinant baculoviruses (Bac-SB-T2CE, BacT2hEA/w, Bac-SB-T2luc/w and Bac-SB-T2hEA/w, respectively) following the instructions of Bac-To-Bac system (Invitrogen). The viruses were propagated by infecting Sf-9 cells and stored according to the standard protocols. For in vivo administration, the virus was concentrated by sucrose-cushioned $(25 \%, \mathrm{w} / \mathrm{v})$ ultracentrifugation and resuspended in PBS ( $\mathrm{pH}$ 6.2). The virus titer was determined by end-point dilution assay and is expressed as p.f.u. per milliliter.

\section{Plasmid transfection and flow cytometry}

HEK293 cells were transfected with the plasmids ( $3 \mu \mathrm{g}$ per $5 \times 10^{5}$ cells) using the lipofectamine 2000 reagent (Invitrogen). After transfection, the cells continued to be cultured and passaged every 3 days. Upon subculture, some cells were measured by a flow cytometer (FACSCalibur, BD Biosciences, San Jose, CA, USA) for the percentage of cells emitting fluorescence (\% of GFP + cells) and mean FI. The total FI was calculated by multiplying the number of GFP + cells and mean FI and is expressed in a.u.

\section{Virus transduction and analysis}

HEK293 cells were transduced with the hybrid SB baculovirus as described $^{47}$ at a multiplicity of infection of 100 . The supernatant was collected at different times for the hEA measurement using the human endostatin Duoset ELISA kit (R\&D Systems, Minneapolis, MN, USA). Alternatively, the cells were harvested for fluorescence microscopy, flow cytometry, PCR or FISH analyses.

\section{PCR and FISH}

For PCR, the template DNA was extracted from the cells or from the purified baculovirus using the Blood \& Tissue Extraction Mini Kit (Viogen, Taipei, Taiwan). The primers were designed to probe the CMV-egfp junction (encompassing the $3^{\prime}$ terminus of CMV-IE promoter and $5^{\prime}$ terminus of egfp) or gapdh as follows: CMV-egfp forward, 5'-CGGGA CTITCCAAAATGTCGTA-3' and CMV-egfp reverse, $5^{\prime}$-TGTTCTGCTGGTAGTG GTCG-3'; gapdh forward, $5^{\prime}$-GTCTCCTCTGACTTCAACAGCG-3' and gapdh reverse, $5^{\prime}$-ACCACCCTGTTGCTGTAGCCAA- $3^{\prime}$. Two pairs of primers specific to two fragments on the baculoviral genome (BM3 and BM4) were designed as described. ${ }^{48}$ PCRs were performed using $100 \mathrm{ng}$ of genomic DNA and the PCR products were subjected to $0.8 \%$ agarose gel electrophoresis. $^{48}$

The FISH experiments were performed as described with minor modifications. ${ }^{20}$ The mitotic cells were prepared at 24 d.p.t. by colcemid $\left(10 \mu \mathrm{g} \mathrm{ml}^{-1}\right.$, Sigma) treatment for $2 \mathrm{~h}$, transferred to microfuge tubes, incubated in the hypotonic $\mathrm{KCl}$ solution $(0.075 \mathrm{M})$ at $37^{\circ} \mathrm{C}$ for $20 \mathrm{~min}$ and fixed in methanol/acetic acid (3:1). After fixation, the cells were dropped onto slides and incubated at $60^{\circ} \mathrm{C}$ overnight. Subsequent hybridization and 4',6-diamidino-2-phenyl-indole staining were performed as described. The data are representative of 150 metaphases.

\section{Tumor models and virus injection}

All animal experiments were performed in compliance with the Guide for the Care and Use of Laboratory Animals (National Science Council, Taiwan). Mouse prostate tumor grafts were created by subcutaneous injection of mouse TRAMP-C1 cells into the dorsal side of 6-week-old C57BL/6J mice $\left(1 \times 10^{6}\right.$ cells per mouse). To establish the ovarian tumor xenografts, human ovarian cancer cells SKOV3 $\left(1 \times 10^{6}\right.$ cells per mouse) were mixed 
with PBS and injected subcutaneously into the back of BALB/c nu/nu mice. The width $(W)$ and length $(L)$ of the tumors were measured twice a week and the volume $(V)$ was calculated by the formula: $V\left(\mathrm{~mm}^{3}\right)=L \times W^{2} \times(\pi / 6)$. When the tumor volume reached $\approx 70 \mathrm{~mm}^{3}$, the mice were randomly grouped and the concentrated baculovirus $\left(3 \times 10^{9}\right.$ p.f.u. per mouse) or PBS $(70 \mu \mathrm{l})$ was injected into the tumor followed by two more injections 3 days apart. The mice were killed and recorded as dead when the tumor volume reached $500 \mathrm{~mm}^{3}$.

\section{Tumor section and immunohistochemical staining}

The tumor specimens were removed from the mice when they reached $200 \mathrm{~mm}^{3}$ in volume. The cryostat sections $(10 \mu \mathrm{m}$ thick) were fixed in methanol, washed and blocked with the blocking buffer (0.1\% Tween-20, $0.1 \mathrm{~g} \mathrm{ml}^{-1}$ bovine serum albumin, $1 \%$ goat serum in PBS, $\left.\mathrm{pH} 7.4\right)$ for $30 \mathrm{~min}$, followed by incubation with the rat anti-mouse CD31 MAb (1:200 dilution, BD Biosciences) at $4{ }^{\circ} \mathrm{C}$ overnight. After three washes, the sections were incubated with Alexa 594-conjugated goat anti-rat IgG (1:200 dilution, Invitrogen) for $1 \mathrm{~h}$ at room temperature. The sections were examined using the confocal microscope and the images were captured. The microvessel density was calculated based on five random fields within the tumor area using Image Pro Plus 6.0 (Media Cybernetics, Silver Spring, $M D$, USA) by dividing the CD31-positive pixels by the total pixels.

\section{Statistical analyses}

The survival curves and median survival were analyzed by the Kaplan-Meier survival test. The log-rank test was used to assess differences among groups for the survival data. For other comparisons, statistical significance was evaluated by Student's $t$-tests.

\section{CONFLICT OF INTEREST}

The authors declare no conflict of interest.

\section{ACKNOWLEDGEMENTS}

We acknowledge the support from the National Tsing Hua University Booster Program (99N2544E1, 98N2901E1 and 97N2511E1), National Science Counci (NSC 97-2627-B-007-014 and NSC 98-2627-B-007-006) and VTY Joint Research Program, Tsou's Foundation (VGHUST98-P5-17), Taiwan.

\section{REFERENCES}

1 Folkman J. Angiogenesis: an organizing principle for drug discovery? Nat Rev Drug Discov 2007; 6: 273-286.

2 Samaranayake H, Maatta A-M, Pikkarainen J, Yla-Herttuala S. Future prospects and challenges of antiangiogenic cancer gene therapy. Hum Gene Ther 2010; 21: 1-16.

3 Thomas CE, Ehrhardt A, Kay MA. Progress and problems with the use of viral vectors for gene therapy. Nat Rev Genet 2003; 4: 346-358.

4 Raikwar SP, Temm CJ, Raikwar NS, Kao C, Molitoris BA, Gardner TA. Adenoviral vectors expressing human endostatin-angiostatin and soluble Tie2: enhanced suppression of tumor growth and antiangiogenic effects in a prostate tumor model. Mol Ther 2005; 12: 1091-1100.

5 Tysome JR, Briat A, Alusi G, Cao F, Gao D, Yu J et al. Lister strain of vaccinia virus armed with endostatin-angiostatin fusion gene as a novel therapeutic agent for human pancreatic cancer. Gene Therapy 2009; 16: 1223-1233.

6 Verma IM, Weitzman MD. Gene therapy: twenty-first century medicine. Ann Rev Biochem 2005; 74: 711-738.

7 Chen $C-Y$, Lin $C-Y$, Chen G-Y, Hu Y-C. Baculovirus as a gene delivery vector: recent understandings of molecular alterations in transduced cells and latest applications. Biotechnol Adv 2011; e-pub ahead of print; doi: 10.1016/j.biotechadv. 2011.04.004.

8 Madhan S, Prabakaran M, Kwang J. Baculovirus as vaccine vectors. Curr Gene Ther 2010; 10: 201-213.

9 Kost TA, Condreay JP, Ames RS. Baculovirus gene delivery: a flexible assay development tool. Curr Gene Ther 2010; 10: 168-173.

10 Liu CY-Y, Chen H-Z, Chao Y-C. Maximizing baculovirus-mediated foreign proteins expression in mammalian cells. Curr Gene Ther 2010; 10: 232-241.

11 Lin C-Y, Lu C-H, Luo W-Y, Chang Y-H, Sung L-Y, Chiu H-Y et al. Baculovirus as a gene delivery vector for cartilage and bone tissue engineering. Curr Gene Ther 2010; 10: 242-254.
12 Airenne KJ, Makkonen K-E, Mähönen AJ, Ylä-Herttuala S. In vivo application and tracking of baculovirus. Curr Gene Ther 2010; 10: 187-194.

13 Kitajima M, Abe T, Miyano-Kurosaki N, Taniguchi M, Nakayama T, Takaku H. Induction of natural killer cell-dependent antitumor immunity by the Autographa californica Multiple Nuclear Polyhedrosis Virus. Mol Ther 2008; 16: 261-268.

14 Wang S, Balasundaram G. Potential cancer gene therapy by baculoviral transduction. Curr Gene Ther 2010; 10: 214-225.

15 Wu C, Lin J, Hong M, Choudhury Y, Balani P, Leung D et al. Combinatorial control of suicide gene expression by tissue-specific promoter and microRNA regulation for cancer therapy. Mol Ther 2009; 17: 2058-2066.

16 Balani P, Boulaire J, Zhao Y, Zeng JM, Lin JK, Wang S. High mobility group box2 promoter-controlled suicide gene expression enables targeted glioblastoma treatment. Mol Ther 2009; 17: 1003-1011.

17 Luo W-Y, Shih, Y-S Lo, W-H, Chen H-R, Wang S-C et al. Baculovirus vectors for antiangiogenesis-based cancer gene therapy. Cancer Gene Ther 2011; 18: 637-645.

18 Ponnazhagan S, Mahendra G, Kumar S, Shaw DR, Stockard CR, Grizzle WE et al. Adeno-associated virus 2-mediated antiangiogenic cancer gene therapy: long-term efficacy of a vector encoding angiostatin and endostatin over vectors encoding a single factor. Cancer Res 2004; 64: 1781-1787.

19 Kost TA, Condreay JP, Jarvis DL. Baculovirus as versatile vectors for protein expression in insect and mammalian cells. Nat Biotechnol 2005; 23: 567-575.

20 Chen $\mathrm{C}-\mathrm{Y}$, Wu H-H, Chen C-P, Chern S-R, Hwang S-M, Huang S-F et al. Biosafety assessment of human mesenchymal stem cells engineered by hybrid baculovirus vectors. Mol Pharm 2011; e-pub ahead of print; doi: 10.1021/mp100368d.

21 Lufino MMP, Edser PAH, Wade-Martins R. Advances in high-capacity extrachromosomal vector technology: episomal maintenance, vector delivery, and transgene expression. Mol Ther 2008; 16: 1525-1538.

22 Lo W-H, Hwang S-M, Chuang C-K, Chen C-Y, Hu Y-C. Development of a hybrid baculoviral vector for sustained transgene expression. Mol Ther 2009; 17: 658-666.

23 Shan L, Wang L-Y, Yin J, Zhong P, Zhong J. An OriP/EBNA-1-based baculovirus vector with prolonged and enhanced transgene expression. J Gene Med 2006; 8: 1400-1406.

24 Wang C-Y, Wang S. Adeno-associated virus inverted terminal repeats improve neuronal transgene expression mediated by baculoviral vectors in rat brain. Hum Gene Ther 2005; 16: 1219-1226.

25 Hackett PB, Largaespada DA, Cooper LN. A transposon and transposase system for human application. Mol Ther 2010; 18: 674-683.

26 Yant SR, Ehrhardt A, Mikkelsen JG, Meuse L, Pham T, Kay MA. Transposition from a gutless adeno-transposon vector stabilizes transgene expression in vivo. Nat Biotechnol 2002; 20: 999-1005.

27 Bowers WJ, Mastrangelo MA, Howard DF, Southerland HA, Maguire-Zeiss KA, Federoff $\mathrm{HJ}$. Neuronal precursor-restricted transduction via in utero CNS gene delivery of a novel bipartite HSV amplicon/transposase hybrid vector. Mol Ther 2006; 13: 580-588.

28 Chen H-C, Lee H-P, Ho Y-C, Sung M-L, Hu Y-C. Combination of baculovirusmediated gene transfer and rotating-shaft bioreactor for cartilage tissue engineering. Biomaterials 2006; 27: 3154-3162.

29 Wang $\mathrm{C}-\mathrm{Y}$, Wang S. Astrocytic expression of transgene in the rat brain mediated by baculovirus vectors containing an astrocyte-specific promoter. Gene Therapy 2006; 13: 1447-1456.

30 Zeng J, Du J, Zhao Y, Palanisamy N, Wang S. Baculoviral vector-mediated transient and stable transgene expression in human embryonic stem cells. Stem Cells 2007; 25: $1055-1061$.

31 Krepple F, Kochanek S. Long-term transgene expression in proliferating cells mediated by episomally maintained high capacity adenovirus vectors. J Virol 2004; 78: 9-22.

32 Ivics Z, Katzer A, Stuwe EE, Fiedler D, Knespel S, Izsvak Z. Targeted Sleeping Beauty transposition in human cells. Mol Ther 2007; 15: 1137-1144.

$33 \mathrm{Hsu}$ C-S, Ho Y-C, Wang K-C, Hu Y-C. Investigation of optimal transduction conditions for baculovirus-mediated gene delivery into mammalian cells. Biotechnol Bioeng 2004; 88: 42-51.

34 Huang X, Guo H, Tammana S, Jung YC, Mellgren E, Bassi P et al. Gene transfer efficiency and genome-wide integration profiling of Sleeping Beauty, Tol2, and piggyBac transposons in human primary T cells. Mol Ther 2010; 18 1803-1813.

35 Mates L, Chuah MKL, Belay E, Jerchow B, Manoj N, Acosta-Sanchez A et al. Molecular evolution of a novel hyperactive Sleeping Beauty transposase enables robust stable gene transfer in vertebrates. Nat Genet 2009; 41: 753-761.

36 Izsvak Z, Hackett PB, Cooper LN, Ivics Z. Translating Sleeping Beauty transposition into cellular therapies: victories and challenges. Bioessays 2010; 32: 756-767.

37 Abe T, Matsuura Y. Host innate immune responses induced by baculovirus in mammals. Curr Gene Ther 2010; 10: 226-231. 
38 Abe $\mathrm{T}$, Kaname $\mathrm{Y}$, Wen $\mathrm{X}$, Tani $\mathrm{H}$, Moriishi $\mathrm{K}$, Uematsu $\mathrm{S}$ et al. Baculovirus induces type $\mathrm{I}$ interferon production through toll-like receptor-dependent and -independent pathways in a cell-type-specific manner. J Virol 2009; 83: 7629-7640.

39 Chuang $\mathrm{C}-\mathrm{K}$, Wong $\mathrm{T}-\mathrm{H}$, Hwang $\mathrm{S}-\mathrm{M}$, Chang $\mathrm{Y}-\mathrm{H}$, Chen $\mathrm{Y}-\mathrm{H}$, Chiu $\mathrm{Y}-\mathrm{C}$ et al. Baculovirus transduction of mesenchymal stem cells: in vitro responses and in vivo immune responses after cell transplantation. Mol Ther 2009; 17: 889-896.

40 Abe T, Hemmi H, Miyamoto H, Moriishi K, Tamura S, Takaku H et al. Involvement of the toll-like receptor 9 signaling pathway in the induction of innate immunity by baculovirus. J Virol 2005; 79: 2847-2858.

41 Suzuki T, Chang MO, Kitajima M, Takaku H. Baculovirus activates murine dendritic cells and induces non-specific NK cell and T cell immune responses. Cell Immunol 2010; 262: 35-43.

42 Hacein-Bey-Abina S, Garrigue A, Wang GP, Soulier J, Lim A, Morillon E et al. Insertional oncogenesis in 4 patients after retrovirus-mediated gene therapy of SCID-X1. J Clin Invest 2008; 118: 3132-3142.
43 Vink CA, Gaspar HB, Gabriel R, Schmidt M, Mclvor RS, Thrasher AJ et al. Sleeping beauty transposition from nonintegrating lentivirus. Mol Ther 2009; 17: 1197-1204.

44 Quesada AR, Medina MÁ, Alba E. Playing only one instrument may be not enough: limitations and future of the antiangiogenic treatment of cancer. Bioessays 2007; 29: 1159-1168.

45 Huang K-S, Lo W-H, Chung Y-C, Lai Y-K, Chen C-Y, Chou S-T et al. Combination of baculovirus-mediated gene delivery and packed-bed reactor for scalable production of adeno-associated virus. Hum Gene Ther 2007; 18: 1161-1170.

46 Mahonen AJ, Airenne KJ, Purola S, Peltomaa E, Kaikkonen MU, Riekkinen MS et al. Post-transcriptional regulatory element boosts baculovirus-mediated gene expression in vertebrate cells. J Biotechnol 2007; 131: 1-8.

47 Chen G-Y, Shiah H-C, Su H-J, Chen C-Y, Chuang Y-J, Lo W-H et al. Baculovirus transduction of mesenchymal stem cells triggers the toll-like receptor 3 (TLR3) pathway. J Virol 2009; 83: 10548-10556.

48 Merrihew RV, Clay WC, Condreay JP, Witherspoon SM, Dallas WS, Kost TA. Chromosomal integration of transduced recombinant baculovirus DNA in mammalian cells. J Virol 2001; 75: 903-909.

Supplementary Information accompanies the paper on Gene Therapy website (http://www.nature.com/gt) 